\title{
ON CUBIC FORMS PERMITTING COMPOSITION
}

\author{
R. D. SCHAFER ${ }^{1}$
}

Let $A$ be a finite-dimensional separable alternative algebra over a field $F$. Then $A=A_{1} \oplus \cdots \oplus A_{r}$, where each simple ideal $A_{i}$ is central simple over its center $Z_{i}$, and $Z_{i}$ is a separable extension of $F$ of degree $d_{i}$. Take $A_{i}$ to be associative for $i=1, \cdots, s(0 \leqq s \leqq r)$ and not associative otherwise. The algebras $A_{i}(i=1, \cdots, s)$ have dimension $m_{i}^{2}$ over $Z_{i}$, and it is well-known $[1, \S 8.11]$ that the principal norm $n_{i}\left(x_{i}\right)$ for $x_{i}$ in $A_{i}$ is a (homogeneous) form of degree $m_{i} d_{i}$ over $F$ satisfying $n_{i}\left(x_{i} y_{i}\right)=n_{i}\left(x_{i}\right) n_{i}\left(y_{i}\right)$. The Cayley algebras $A_{i}(i=s+1, \cdots, r)$ are of degree $m_{i}=2$ and dimension 8 over $Z_{i}$, and there is similarly a norm $n_{i}\left(x_{i}\right)=N_{Z_{i} / F}\left(n_{A_{i} / Z_{i}}\left(x_{i}\right)\right)$ of degree $m_{i} d_{i}$ $=2 d_{i}$ satisfying $n_{i}\left(x_{i} y_{i}\right)=n_{i}\left(x_{i}\right) n_{i}\left(y_{i}\right)$. Let $x=x_{1}+\cdots+x_{r}$ for $x_{i}$ in $A_{i}$, and define

$$
N(x)=\left[n_{1}\left(x_{1}\right)\right]^{f_{1}} \cdots\left[n_{r}\left(x_{r}\right)\right]^{f_{r}}
$$

for arbitrary positive integers $f_{i}$. Then $N(x)$ is a form of degree $n$ where

$$
n=\sum_{i=1}^{r} f_{i} m_{i} d_{i} \quad\left(m_{i}=2 \text { for } i=s+1, \cdots, r\right) .
$$

Also $N(x)$ permits composition. That is,

$$
N(x y)=N(x) N(y) .
$$

The dimension of $A$ over $F$ is

$$
\operatorname{dim} A=\sum_{i=1}^{s} m_{i}^{2} d_{i}+8 \sum_{i=s+1}^{r} d_{i}
$$

Ignoring the question of inseparability by assuming characteristic $\neq 2$, we may state the principal fact about quadratic forms permitting composition $[2 ; 7 ; 6]$ as follows. Let $A$ be a nonassociative algebra (of possibly infinite dimension) over $F$ of characteristic $\neq 2$, and assume $^{2}$ that $A$ has a unity element 1 . If $N(x)$ is a nondegenerate quadratic form on $A$ permitting composition, then $A$ is a finitedimensional separable alternative algebra over $F$ and $N(x)$ is given by

Presented to the Society April 17, 1959; received by the editors March 14, 1959.

${ }^{1}$ National Science Foundation Senior Postdoctoral Fellow.

2 An easy modification suffices in case $A$ has no unity element $[7$, p. $957 ; 6$, p. 56]. See also the remark at the end of this paper. 
(1) with $n=2$ in (2). This limits the possibilities severely, and by (4) one has the classical restriction: the dimension of $A$ is $1,2,4$, or 8 . Conversely, any such quadratic norm form is nondegenerate.

A plausible conjecture is that any (possibly infinite-dimensional) algebra $A$ with 1 over $F$ of characteristic 0 or $p>n$, on which a nondegenerate ${ }^{3}$ form $N(x)$ of degree $n$ and permitting composition is defined, is a finite-dimensional separable alternative algebra with $N(x)$ given by (1). Then (2) and (4) would give the restrictions on the structure and dimension of $A$.

In this paper we study cubic forms permitting composition, and prove the following

Theorem. Let $A$ be a finite-dimensional nonassociative algebra with 1 over $F$ of characteristic $\neq 2,3$. A necessary and sufficient condition for the existence of a nondegenerate cubic form $N(x)$ on $A$ permitting composition is that $A$ be a separable alternative algebra for which $N(x)$ is given by (1) with $n=3$ in (2); that is, one of:

(i) $F 1 \quad$ with $f_{1}=3$ in (1)),

(ii) a cubic field over $F$,

(iii) a central simple associative algebra of dimension 9 over $F$,

(iv) $F e_{1} \oplus B$ where $B$ is an algebra (with unity element $1-e_{1}$ ) on which a nondegenerate quadratic form permitting composition is defined; that is, one of:

(iv, a) $F e_{1} \oplus F e_{2} \quad$ (with $f_{1}=1, f_{2}=2$ in (1)),

(iv, b) $F e_{1} \oplus F e_{2} \oplus F e_{3}$,

(iv, c) $F e_{1} \oplus Z, Z$ a quadratic field over $F$,

(iv, d) $F e_{1} \oplus Q, \quad Q$ a (generalized) quaternion algebra over $F$,

(iv, e) $F e_{1} \oplus C, \quad C$ a Cayley algebra over $F$.

The possible dimensions for $A$ are 1, 2, 3, 5, and 9.

Sufficiency is immediate, and we are concerned throughout the paper with proving the necessity. Our method (a reduction to traceadmissible algebras) makes essential use in Lemma 2 of the assumed finite dimensionality of $A$.

1. Cubic forms. Let $V$ be a vector space (of possibly infinite dimension) over a field $F$ of characteristic $\neq 2$, 3. A mapping $x \rightarrow N(x)$ of $V$ into $F$ is called a cubic form on $V$ in case $N(\alpha x)=\alpha^{3} N(x)$ for all $\alpha \in F$ and $x \in V$, and

$$
\begin{aligned}
(x, y, z)= & \frac{1}{6}[N(x+y+z)-N(x+y)-N(x+z)-N(y+z) \\
& +N(x)+N(y)+N(z)]
\end{aligned}
$$

${ }^{3}$ For the definition, see footnote 4 . 
is trilinear. Then $N(x)=(x, x, x)$. We shall say that a symmetric trilinear form $(x, y, z)$ and its associated cubic form are nondegenerate in case $(x, y, z)=0$ for all $y, z$ in $V$ implies $x=0 .{ }^{4}$

Assume that a cubic form $N(x)$ is defined on a nonassociative algebra $A$ over $F$, and that $N(x)$ permits composition: ( $x y, x y, x y)$ $=(x, x, x)(y, y, y)$. We linearize this in $x$ to

$$
\left(x_{1} y, x_{2} y, x_{3} y\right)=\left(x_{1}, x_{2}, x_{3}\right) N(y),
$$

and linearize (5) in $y$ to the fundamental relationship

$$
\begin{gathered}
\left(x_{1} y_{1}, x_{2} y_{2}, x_{3} y_{3}\right)+\left(x_{1} y_{1}, x_{2} y_{3}, x_{3} y_{2}\right)+\left(x_{1} y_{2}, x_{2} y_{1}, x_{3} y_{3}\right) \\
+\left(x_{1} y_{2}, x_{2} y_{3}, x_{3} y_{1}\right)+\left(x_{1} y_{3}, x_{2} y_{1}, x_{3} y_{2}\right)+\left(x_{1} y_{3}, x_{2} y_{2}, x_{3} y_{1}\right) \\
=6\left(x_{1}, x_{2}, x_{3}\right)\left(y_{1}, y_{2}, y_{3}\right) .
\end{gathered}
$$

Clearly (6) is equivalent to (3) when the characteristic is $\neq 2,3$. Also (6) implies

$$
\left(x y_{1}, x y_{2}, x y_{3}\right)=N(x)\left(y_{1}, y_{2}, y_{3}\right) .
$$

Assume that $A$ contains 1 . Define a linear form $x \rightarrow T(x)$ on $A$ by $T(x)=3(x, 1,1)$ and a quadratic form $x \rightarrow Q(x)=3(x, x, 1)$. We derive a number of consequences of (6) for future use. Put $x_{1}=x, x_{2}=y$, $x_{3}=z, y_{1}=a, y_{2}=y_{3}=1$ in (6) to obtain

$$
(x a, y, z)+(x, y a, z)+(x, y, z a)=(x, y, z) T(a) .
$$

That is, a right multiplication $R_{a}$ on $A$ leaves $(x, y, z)$ invariant if $T(a)=0$. Symmetrically,

$$
(a x, y, z)+(x, a y, z)+(x, y, a z)=T(a)(x, y, z) .
$$

Then (8) and (9) imply

$$
([x, a], y, z)+(x,[y, a], z)+(x, y,[z, a])=0
$$

so that $R_{a}-L_{a}$ leaves $(x, y, z)$ invariant for every $a$ in $A$.

Replace $x$ by $x^{2}$ and put $a=x$ in (8) and (9) to obtain

$$
\left(x^{2} x, y, z\right)+\left(x^{2}, y x, z\right)+\left(x^{2}, y, z x\right)=\left(x^{2}, y, z\right) T(x)
$$

and

$$
\left(x x^{2}, y, z\right)+\left(x^{2}, x y, z\right)+\left(x^{2}, y, x z\right)=T(x)\left(x^{2}, y, z\right) .
$$

Replace $x$ by $x^{2}$ and put $a=x^{2}$ in (9) to obtain

4 More generally, if $F$ has characteristic 0 or $p>n$, and if $N(x)$ is a form of degree $n$ with associated $n$-linear form $\left(x_{1}, x_{2}, \cdots, x_{n}\right)$ obtained by polarization, we shall say that $\left(x_{1}, x_{2}, \cdots, x_{n}\right)$ and $N(x)=(x, x, \cdots, x)$ are nondegenerate in case $\left(x_{1}, x_{2}, \cdots, x_{n}\right)=0$ for all $x_{2}, \cdots, x_{n}$ in $V$ implies $x_{1}=0$. 


$$
\left(x^{2} x^{2}, y, z\right)+\left(x^{2}, x^{2} y, z\right)+\left(x^{2}, y, x^{2} z\right)=T\left(x^{2}\right)\left(x^{2}, y, z\right) .
$$

Put $z=1$ in (9) and rewrite to obtain

$$
(x y, z, 1)+(y, x z, 1)+(y, z, x)=T(x)(y, z, 1) .
$$

Now $z=1$ in (14) implies

$$
T(x y)=T(x) T(y)-6(x, y, 1),
$$

so that, in particular,

$$
T\left(x^{2}\right)=[T(x)]^{2}-2 Q(x) .
$$

Also (15) implies

$$
T(x y)=T(y x) \quad \text { for all } x, y \text { in } A .
$$

Moreover, (15) and (14) imply

$$
T((x y) z)=T(x(y z)) \quad \text { for all } x, y, z \text { in } A .
$$

For $T((x y) z)-T(x(y z))=T(x y) T(z)-6(x y, z, 1)-T(x) T(y z)$ $+6(x, y z, 1)=-6(x, y, 1) T(z)-6(x y, z, 1)+6 T(x)(y, z, 1)+6(x, y z, 1)$ $=6[-(x, y, 1) T(z)+(y, x z, 1)+(y, z, x)+(x, y z, 1)]$. Using the relationship which results from interchange of $x$ and $z$ in (14), we have $T((x y) z)-T(x(y z))=6[([x, z], y, 1)+(x,[y, z], 1)]=-6(x, y,[1, z])$ $=0$ by $(10)$.

Next put $x_{1}=x^{2}, x_{2}=x_{3}=x, y_{1}=y, y_{2}=z, y_{3}=1$ in (6); using (7), we have

(19) $\left(x^{2} y, x z, x\right)+\left(x^{2} z, x y, x\right)=N(x) T(x)(1, y, z)-N(x)(x, y, z)$.

Also, putting $x_{1}=y_{1}=y_{2}=x, x_{2}=y, x_{3}=z, y_{3}=1$ in (6) we have

$$
\left(x^{2}, y x, z\right)+\left(x^{2}, y, z x\right)=Q(x)(x, y, z)-N(x)(1, y, z)
$$

by (5). Symmetrically, we have

$$
\left(x^{2}, x y, z\right)+\left(x^{2}, y, x z\right)=Q(x)(x, y, z)-N(x)(1, y, z) .
$$

Put $x_{1}=y_{1}=x, x_{2}=x^{2}, x_{3}=1, y_{2}=y, y_{3}=z$ in (6) to obtain

$$
\begin{aligned}
\left(x^{2}, x^{2} y, z\right)+ & \left(x^{2}, x^{2}, z, y\right)+\left(x y, x^{2} x, z\right)+\left(x y, x^{2} z, x\right) \\
& +\left(x z, x^{2} x, y\right)+\left(x z, x^{2} y, x\right)=6\left(x, x^{2}, 1\right)(x, y, z) .
\end{aligned}
$$

Finally put $x_{1}=x_{2}=x, y_{1}=y_{2}=y, x_{3}=y_{3}=1$ in (6) to obtain

$$
Q(x y)+6(x y, x, y)=Q(x) Q(y) .
$$

Assume henceforth that $N(x)$ is nondegenerate. Then (3) implies $N(1)=1$, and therefore $T(1)=Q(1)=3$. Also $x^{3}$ is uniquely defined $\left(x^{2} x=x x^{2}\right)$ for any $x$ in $A$, and 


$$
x^{3}-T(x) x^{2}+Q(x) x-N(x) 1=0 ;
$$

that is, $A$ is a cubic algebra. For (11) and (20) imply $\left(x^{2} x-T(x) x^{2}\right.$ $+Q(x) x-N(x) 1, y, z)=0$ for all $y, z$ in $A$, or $x^{2} x-T(x) x^{2}+Q(x) x$ $-N(x) 1=0$. Similarly (12) and (21) imply $x x^{2}-T(x) x^{2}+Q(x) x$ $-N(x) 1=0$, so that $x^{2} x=x x^{2}$ and (24) holds. Also

$$
x^{2} x^{2}=x^{3} x\left(=x x^{3}\right)
$$

for $x$ in $A$.

For (13) and (22) imply $\left(x^{2} x^{2}-T\left(x^{2}\right) x^{2}, y, z\right)=\left(x y, z, x^{3}\right)+\left(y, x z, x^{3}\right)$ $+\left(x^{2} z, x y, x\right)+\left(x^{2} y, x, x z\right)-6\left(x, x^{2}, 1\right)(x, y, z)$. Now $\left(x y, z, x^{3}\right)$ $+\left(y, x z, x^{3}\right)=\left(Q(x) x^{2}-N(x) x, y, z\right)$ by (24), (21), (8), and (14). Also $6\left(x, x^{2}, 1\right)=Q(x) T(x)-3 N(x)$ by (15) and (24). Hence (16) implies

$$
x^{2} x^{2}=\left\{[T(x)]^{2}-Q(x)\right\} x^{2}+[N(x)-Q(x) T(x)] x+N(x) T(x) 1 .
$$

Then (24) and (26) imply (25). Hence $A$ is power-associative by

Lemma 1. Any cubic algebra in which (25) is satisfied is powerassociative.

Proof. Let $x^{2} x=x x^{2}=\alpha x^{2}+\beta x+\gamma 1$, and define $x^{i}=x^{i-1} x=\alpha_{i} x^{2}$ $+\beta_{i} x+\gamma_{i} 1$. It is well-known that any quadratic algebra is powerassociative, so we may assume that $x^{2}, x, 1$ are linearly independent. Then $\alpha_{1}=0, \beta_{1}=1, \gamma_{1}=0, \alpha_{i}=\alpha_{i-1} \alpha+\beta_{i-1}, \beta_{i}=\alpha_{i-1} \beta+\gamma_{i-1}, \gamma_{i}=\alpha_{i-1} \gamma$, and it is readily established by induction on $j$ and use of (25) that $x^{i} x^{i}=x^{i+j}$.

2. Trace-admissibility. Under the assumptions of $\S 1$ we have seen that $A$ is a power-associative algebra with 1 on which a linear form $T(x)$ is defined satisfying (17) and (18). Then the bilinear form $B(x, y)=T(x y)$ is an admissible trace function [4] for $A: B(x, y)$ $=B(y, x), B(x y, z)=B(x, y z), B(e, e) \neq 0$ for any idempotent $e$, $B(x, y)=0$ if $x y$ is nilpotent. For we can prove $T(e) \neq 0$ for any idempotent $e$ and $T(z)=0$ for any nilpotent element $z$.

Assume $e \neq 1$ since $T(1)=3 \neq 0$. Then $e$ and 1 are obviously linearly independent and

$$
Q(e)-T(e)+1=0, \quad N(e)=0
$$

if $e \neq 1$,

by (24). Then (16) and (27) imply

$$
\text { either } T(e)=1 \text { or } T(e)=2
$$

if $e \neq 1$.

Now $z=0$ implies $T(z)=N(z)=0$. Then $z^{r}=0, r>1$, implies $N(z)=0$ by (3). If $z^{2}=0$, but $z \neq 0$, then (24) implies $Q(z)=0$ so that $T(z)=0$ by (16). We may assume therefore that $z^{2} \neq 0$, and let $r$ be the least exponent such that $z^{r}=0\left(z^{r-1} \neq 0, r>2\right)$. Then $z^{r-1}$ and $z^{r-2}$ are 
obviously linearly independent, so that (24) implies $T(z)=Q(z)=0$ (giving $z^{3}=0$ ).

Also $B(x, y)$ is nondegenerate on $A$. For, if $B(x, y)=0$ for every $y$ in $A$, then $B(x, 1)=T(x)=0$. Also $B(x, y z)=0$ for all $y, z$ in $A$, implying $B(x y, z)=B(x, y) T(z)-6(x y, z, 1)=-6(x y, z, 1)=0$ by (15). Interchange $y$ and $z$ to obtain $(x z, y, 1)=0$, so that $(x, y, z)=0$ for all $y, z$ in $A$ by (14). Then $x=0$.

If we now assume that $A$ is of finite dimension over $F$, we can apply $[4]^{5}$ to obtain

Lemma 2. Let $A$ be a finite-dimensional nonassociative algebra with 1 over $F$ of characteristic $\neq 2,3$. There is a nondegenerate cubic form $N(x)$ on $A$ permitting composition only if $A$ is a separable algebra $A=A_{1} \oplus \cdots \oplus A_{r}$ in which each simple ideal $A_{i}$ is one of the following: a (commutative) Jordan algebra, a quasiassociative algebra [3, Chapter $\mathrm{V}]$, or a flexible quadratic algebra (with the attached commutative algebra $A_{i}^{+}$a simple Jordan algebra of degree 2 ).

We shall first sharpen this result considerably in case $r>1$. Write $x=x_{1}+\cdots+x_{r}, x_{i}$ in $A_{i}$, and $1=e_{1}+\cdots+e_{r}$ where $e_{i}$ is the unity element of $A_{i}\left(e_{i} \neq 1\right.$ in case $\left.r>1\right)$. Consider $N_{i}\left(x_{i}\right)=N\left(x_{i}-e_{i}+1\right)$. Then $N(x)=N_{1}\left(x_{1}\right) \cdots N_{r}\left(x_{r}\right)$ and

$$
N_{i}\left(x_{i} y_{i}\right)=N_{i}\left(x_{i}\right) N_{i}\left(y_{i}\right) .
$$

For $\quad N_{1}\left(x_{1}\right) \cdots N_{r}\left(x_{r}\right)=N\left(x_{1}-e_{1}+1\right) \cdots N\left(x_{r}-e_{r}+1\right)$ $=N\left(\left(x_{1}-e_{1}+1\right) \cdots\left(x_{r}-e_{r}+1\right)\right) \quad$ (associative product!) $=N\left(x_{1}+\cdots+x_{r}-\left(e_{1}+\cdots+e_{r}\right)+1\right)=N\left(x_{1}+\cdots+x_{r}\right)$, while $N_{i}\left(x_{i} y_{i}\right)=N\left(x_{i} y_{i}-e_{i}+1\right)=N\left(\left(x_{i}-e_{i}+1\right)\left(y_{i}-e_{i}+1\right)\right)=N_{i}\left(x_{i}\right) N_{i}\left(y_{i}\right)$.

Now

$$
\begin{aligned}
N_{i}\left(x_{i}\right)= & \left(x_{i}-e_{i}+1, x_{i}-e_{i}+1, x_{i}-e_{i}+1\right) \\
= & N\left(x_{i}\right)-3\left(x_{i}, x_{i}, e_{i}\right)+Q\left(x_{i}\right)+3\left(x_{i}, e_{i}, e_{i}\right)+Q\left(e_{i}\right) \\
& +T\left(x_{i}\right)-T\left(e_{i}\right)-N\left(e_{i}\right)+N(1)-6\left(x_{i}, e_{i}, 1\right) .
\end{aligned}
$$

If $e_{i} \neq 1$, then $N\left(x_{i}\right)=N\left(x_{i} e_{i}\right)=N\left(x_{i}\right) N\left(e_{i}\right)=0$ and $Q\left(e_{i}\right)-T\left(e_{i}\right)$ $+N(1)=0$ by (27). Put $a=e_{i}, x=y=x_{i}, z=1$ in (8) and $x=x_{i}$, $y=e_{i}$ in (23) to obtain $2 Q\left(x_{i}\right)+3\left(x_{i}, e_{i}, e_{i}\right)=Q\left(x_{i}\right) T\left(e_{i}\right)$ and $Q\left(x_{i}\right)$ $+6\left(x_{i}, e_{i}, e_{i}\right)=Q\left(x_{i}\right) Q\left(e_{i}\right)$. Then (27) implies

$$
2 Q\left(x_{i}\right)=Q\left(x_{i}\right) T\left(e_{i}\right), \quad\left(x_{i}, e_{i}, e_{i}\right)=0
$$

if $e_{i} \neq 1$.

${ }^{5}$ It is not necessary to assume characteristic $\not 5$. One can see from [8] that this restriction in [4] may be replaced by power-associativity in any scalar extension $A_{K}$ of $A$. But our identity (6), being linear in each argument, and being equivalent to (3), insures that all of our results hold for $A_{K}$ where $K$ is any extension of $F$. In particular, since $A$ is semisimple by [4], we know that $A_{K}$ is semisimple; that is, $A$ is separable. 
Put $a=x_{i}, x=y=e_{i}, z=1$ in (8) and $x=x_{i}, y=e_{i}$ in (15) to obtain $6\left(x_{i}, e_{i}, 1\right)+3\left(e_{i}, e_{i}, x\right)=Q\left(e_{i}\right) T\left(x_{i}\right)$ and $T\left(x_{i}\right)=T\left(x_{i}\right) T\left(e_{i}\right)-6\left(x_{i}, e_{i}, 1\right)$. Then (27) implies

$$
6\left(x_{i}, e_{i}, 1\right)=Q\left(e_{i}\right) T\left(x_{i}\right), \quad\left(e_{i}, e_{i}, x_{i}\right)=0 \quad \text { if } e_{i} \neq 1 .
$$

Hence $N_{i}\left(x_{i}\right)=Q\left(x_{i}\right)+T\left(x_{i}\right)-Q\left(e_{i}\right) T\left(x_{i}\right)$ if $e_{i} \neq 1$. By (28) there are two cases. If $T\left(e_{i}\right)=1$, then $Q\left(x_{i}\right)=0$ by $(30)$ and $N_{i}\left(x_{i}\right)=T\left(x_{i}\right)$. If $T\left(e_{i}\right)=2$, then $Q\left(e_{i}\right)=1$ by $(27)$ and $N_{i}\left(x_{i}\right)=Q\left(x_{i}\right)$. Thus, if $r>1$, we can order the simple $A_{i}$ so that $T\left(e_{i}\right)=1$ for $i=1, \cdots, t, T\left(e_{i}\right)=2$ for $i=t+1, \cdots, r(0 \leqq t \leqq r)$. Then $N(x)=N_{1}\left(x_{1}\right) \cdots N_{r}\left(x_{r}\right)$ $=T\left(x_{1}\right) \cdots T\left(x_{t}\right) Q\left(x_{t+1}\right) \cdots Q\left(x_{r}\right)$ is of degree $t+2(r-t)=2 r-t=3$. The only possibilities are $r=2, t=1$, and $r=t=3$. That is, if $A$ is not simple, then either $A=A_{1} \oplus A_{2}$ with $T\left(e_{1}\right)=1, N_{1}\left(x_{1}\right)=T\left(x_{1}\right), T\left(e_{2}\right)$ $=2, Q\left(e_{2}\right)=1, \quad N_{2}\left(x_{2}\right)=Q\left(x_{2}\right), \quad$ or $A=A_{1} \oplus A_{2} \oplus A_{3}$ with $T\left(e_{i}\right)=1$, $N_{i}\left(x_{i}\right)=T\left(x_{i}\right)$ for $i=1,2,3$.

Consider the situation where $N_{i}\left(x_{i}\right)=T\left(x_{i}\right)$. By (29) we have $B\left(x_{i}, y\right)=T\left(x_{i} y\right)=T\left(x_{i} y_{i}\right)=T\left(x_{i}\right) T\left(y_{i}\right)=T\left(x_{i}\right) T\left(e_{i} y\right)=T\left(x_{i}\right) B\left(e_{i}, y\right)$ $=B\left(T\left(x_{i}\right) e_{i}, y\right)$ for every $y \in A$. Hence $x_{i}=T\left(x_{i}\right) e_{i}$ for every $x_{i}$ in $A_{i}$, or $A_{i}=F e_{i}$. Thus, if $A$ is not simple, we have case (iv) of our Theorem as soon as we verify that $N_{2}\left(x_{2}\right)=Q\left(x_{2}\right)$ is nondegenerate on $A_{2}$. Suppose that

$$
\left(x_{2}, y_{2}\right)=\frac{1}{2}\left[Q\left(x_{2}+y_{2}\right)-Q\left(x_{2}\right)-Q\left(y_{2}\right)\right]=3\left(x_{2}, y_{2}, 1\right)=0
$$

for all $y_{2} \in A_{2}$. Then $0=2\left(x_{2}, e_{2}\right)=6\left(x_{2}, e_{2}, 1\right)=Q\left(e_{2}\right) T\left(x_{2}\right)=T\left(x_{2}\right)$ by (31), and $B\left(x_{2}, \quad y\right)=T\left(x_{2} y\right)=T\left(x_{2} y_{2}\right)=T\left(x_{2}\right) T\left(y_{2}\right)-6\left(x_{2}, \quad y_{2}, \quad 1\right)$ $=-2\left(x_{2}, y_{2}\right)=0$ for all $y \in A$ by (15), implying $x_{2}=0$.

3. Simple algebras. We are left with the case where $A$ is simple. Let $K$ be a splitting field for $A$. Since all of our results are valid ${ }^{6}$ for $A_{K}$, we know that one of the following is true: $A_{K}$ is simple (implying that $A$ is central simple over $F$ ), $A_{K}=K e_{1} \oplus S$ (implying, since the simple components of $A_{K}$ are all isomorphic, that $S=K e_{2}$ and that $A$ is a quadratic field over $F$, a possibility to be eliminated in Lemma 3 ), or $A_{K}=K e_{1} \oplus K e_{2} \oplus K e_{3}$ (implying that $A$ is a cubic field over $F$, which is case (ii) of the Theorem).

Suppose that $A$ is central simple, and that $K$ is the algebraic closure of $F$. Since each element of $A_{K}$ satisfies an equation of degree 3 (or lower) with coefficients in $K$, Lemma 2 implies that $A_{K}$ is one of: (a) a split central simple (commutative) Jordan algebra of degree 3 (dimension $6,9,15$, or 27 ); (b) a split central simple quasiassociative

- See footnote 5 . 
algebra of degree 3 ; (c) a split central simple flexible quadratic algebra (with the attached commutative algebra $A_{K}^{+}$a split central simple Jordan algebra of degree 2 , dimension $\geqq 3$ ); (d) $K 1$ (implying $A=F 1$, case (i) of the Theorem).

In (a) and (b) we may represent the elements of $A_{K}$ by certain $3 \times 3$ matrices; in each case the set $S$ of all $3 \times 3$ symmetric matrices with elements in $K$ is included. Multiplication in $A_{K}$ is defined by $x y=\lambda x \circ y+(1-\lambda) y \circ x$ for some $\lambda \in K(\lambda=1 / 2$ for the algebras (a)) where $x \circ y$ denotes the ordinary matrix product. Powers of elements of $S$ coincide with ordinary matrix powers. Consider

$$
e=\left(\begin{array}{lll}
1 & 0 & 0 \\
0 & 0 & 0 \\
0 & 0 & 1
\end{array}\right), \quad z=\left(\begin{array}{lll}
0 & 1 & 0 \\
1 & 0 & 0 \\
0 & 0 & 0
\end{array}\right)
$$

in $S$. Now $e \neq 1$ is an idempotent, and $x=e(z-e)=e z-e=\lambda e \circ z$ $+(1-\lambda) z \circ e-e$ is the matrix

$$
x=\left(\begin{array}{ccr}
-1 & \lambda & 0 \\
1-\lambda & 0 & 0 \\
0 & 0 & -1
\end{array}\right) .
$$

But $x$ satisfies the equation $x^{3}+2 x^{2}+\left(\lambda^{2}-\lambda+1\right) x+\left(\lambda^{2}-\lambda\right) 1=0$ and, if $\lambda \neq 0,1$, no equation of lower degree. Hence $N(x)=\lambda-\lambda^{2}=N(e(z-e))$ $=N(e) N(z-e)=0$ by (27), a contradiction unless $\lambda=0$ or $\lambda=1$. This eliminates (a) and leaves for (b) only an associative algebra. For the corresponding algebras $A$ we have case (iii) of our Theorem.

Quadratic fields and possibility (c) above are eliminated in

Lemma 3. A nondegenerate cubic form $N(x)$ permitting composition cannot be defined on a quadratic field $A$ over $F$ of characteristic $\neq 2,3$, or on an algebra $A$ over $F$ for which the attached commutative algebra $A^{+}$ is a central simple Jordan algebra of degree 2.

Proof. For $x$ in $A$ satisfies $x^{2}=t(x) x-n(x) 1$ with $t(x), n(x)$ in $F$, $t(\alpha 1)=2 \alpha, n(\alpha 1)=\alpha^{2}$. Also $A=F 1+M$ where $M$ consists of all $x_{0}$ satisfying $t\left(x_{0}\right)=0$. If $A$ is a quadratic field, $M$ contains $u_{0}$ with $u_{0}^{2}=\gamma 1=-n\left(u_{0}\right) 1, \gamma$ a nonsquare in $F$. In the other case, there is a nondegenerate quadratic form $f[5, \S 13]$ on $M$ of dimension $\geqq 2$ such that $x_{0}^{2}=f\left(x_{0}\right) 1=-n\left(x_{0}\right) 1$ for $x_{0} \in M$. Now (24) implies $[T(x)-t(x)] x^{2}$ $+[n(x)-Q(x)] x+N(x) 1=0$. Write $L(x)=T(x)-t(x)$. Then $[n(x)-Q(x)+L(x) t(x)] x+[N(x)-L(x) n(x)] 1=0$. Whether or not $x \notin F 1$, we have $Q(x)=n(x)+L(x) t(x)$. Then (16) implies $n(x)$ $=L(x)[t(x)-L(x)]$, so that $n\left(x_{0}\right)=-\left[L\left(x_{0}\right)\right]^{2}$ for all $x_{0} \in M$. Thus 
$\gamma=-n\left(u_{0}\right)=\left[L\left(u_{0}\right)\right]^{2}$, a contradiction, and the nondegenerate quadratic form $f\left(x_{0}\right)=-n\left(x_{0}\right)=\left[L\left(x_{0}\right)\right]^{2}$ is the square of a linear form $L\left(x_{0}\right)$ on $M$, implying that $M$ is 1 -dimensional, a contradiction. This completes the proof of Lemma 3 and of the Theorem.

REMARK. If $A$ does not contain 1, it is possible to pass easily (as in $\left[7\right.$, p. $957 ; 6$, p. 56]) to an isotopic algebra $A^{*}$ with 1 . Briefly: $N(u) \neq 0$ implies by (5) and (7) that $x \rightarrow x a=x R_{a}$ and $x \rightarrow a x=x L_{a}$ are (1-1) for $a=u^{3} / N(u)$. By finite-dimensionality we can define multiplication in $A^{*}$ by $x * y=\left(x R_{a}^{-1}\right)\left(y L_{a}^{-1}\right)$. Then $a^{2}$ is a unity element for $A^{*}$ and $N(x * y)=N(x) N(y)$. Thus, without assuming $1 \in A$, we have $\operatorname{dim} A=1,2,3,5$, or 9 .

\section{REFERENCES}

1. A. A. Albert, Structure of algebras, Amer. Math. Soc. Colloquium Publications, vol. $24,1939$.

2. - Quadratic forms permitting composition, Ann. of Math. vol. 43 (1942) pp. 161-177.

3. - Power-associative rings, Trans. Amer. Math. Soc. vol. 64 (1948) pp. 552-593.

4. - A theory of trace-admissible algebras, Proc. Nat. Acad. Sci. U.S.A. vol. 35 (1949) pp. 317-322.

5. Nathan Jacobson, Structure of alternative and Jordan bimodules, Osaka Math. J. vol. 6 (1954) pp. 1-71.

6. - Composition algebras and their automorphisms, Rend. Circ. Mat. Palermo (2) vol. 7 (1958) pp. 55-80.

7. Irving Kaplansky, Infinite-dimensional quadratic forms admitting composition, Proc. Amer. Math. Soc. vol. 4 (1953) pp. 956-960.

8. L. A. Kokoris, New results on power-associative algebras, Trans. Amer. Math. Soc. vol. 77 (1954) pp. 363-373.

UNiversity of ConNecticut AND Institute for Advanced Study 